Meyler's provides an encyclopaedic survey this new publication is intended to give regular critical reviews of recent reports, starting at January 1975. The editor has chosen a team of contributors who are practising clinicians, mainly British and from the mainland of Europe; their combined efforts are undoubtedly highly successful. This initial volume provides readable, authoritative accounts of all fields of drug usage. There are useful indices to drugs, synonyms and side effects and the references are coded to indicate whether the paper is a critical review, detailed clinical report, etc.

This book can be warmly recommended as a valuable addition to the not insubstantial review literature of drug side effects for reference as an aid to keeping up to date in one's own field, and for pleasurable browsing.

\section{The Specific Treatment of Virus Diseases}

By D. J. Bauer. Pp. 194, illustrated, hard cover. MTP Press, Lancaster, 1977. £8.95.

This is an excellent review of the currently available drugs with specific antiviral activity which have relevance to clinical medicine.

Antiviral drugs have been in clinical use for approximately 15 years. At present, topical therapy is generally more satisfactory than systemic, but one hopes that more effective and less toxic drugs will be developed in the future. Recent work with amantidine hydrochloride suggests that we may be very grateful for this drug in prophylaxis and therapy in any future influenza pandemic.

Many clinicians, particularly ophthalmologists, dermatologists, neurologists and infectious disease specialists, are using antiviral drugs. They should welcome this book for its collected documentation of past experience with the drugs and for the extensive reference list.

It should prove worthwhile reading for the medical undergraduate but it is doubtful whether many students will be in a financial position to possess their own copy.

There are four chapters relating to the treatment of patients with specific virus diseases. As the book is intended fo clinicians, it seems a pity that the author has not taken th\& opportunity to include a little more advice on generad. management and the use of certain drugs with non-specific. activity. This would, perhaps, be more relevant than some of the basic virological information which is readily available elsewhere.

There are few significant errors, but one is worthy of note In the list of preparations of idoxuridine, Herpid (W. BD Pharmaceuticals) is stated to contain $0.5 \%$ idoxuridine in dimethylsulphoxide. This should be $5.0 \%$ idoxuridine, an as the other listed preparations are for ophthalmic use, fै could have serious consequences particularly as dimethylsulphoxide is toxic in the eye.

The author is to be congratulated for producing a ver $\overrightarrow{\underline{\Sigma}}$ readable and informative review of a field in which significang advances are continuing to come from his own laboratory.

It is recommended to all clinicians who are concerned wito the management of viral illness, and that is surely the majority.

\section{Statistics at Square One}

By T. D. V. Swinscow. Pp. vi +86 , illustrated, soft cover.? British Medical Association, London, 1976. £1.50, \$3.7\$ including postage.

This is an excellent book that fulfils a definite need, parti? cularly for the postgraduate medical student entering th $\vec{\varepsilon}$ bewildering world of statistics during his first researcby appointment. It explains the basic principles of the subject it a way comprehensible to anyone with no more than an 'o" level training in mathematics (and that includes man doctors!) without being patronizing. The whole approact a logical progression from the simplest principles to thes slightly more complicated. The only criticism is that the boro $R^{\circ}$ could have been longer with chapters dealing with advanced statistics. One, therefore, sincerely hopes tha second volume will follow. 